\title{
Redes complexas na extração de características em imagens de peixes
}

\author{
Alexander Scaranti ${ }^{1}$, Ronaldo Bernardi ${ }^{1}$ \\ ${ }^{1}$ Universidade Paulista - UNIP
}

\section{Resumo}

Este trabalho apresenta uma abordagem sobre a análise de imagens utilizando as técnicas de Redes Complexas para a obtenção de similaridades entre imagens. Inicialmente serão extraídas as características da borda das imagens, as quais serão entendidas computacionalmente como matrizes. Através dessas matrizes serão geradas matrizes de adjacências, que serão utilizadas para a verificação das conexões dos vértices (pixels) das imagens. Extraída a matriz de adjacência, será realizada a extração do grau dos vértices para um limiar T e a extração do grau médio dos vértices. Na extração do grau dos vértices cada limiar será aplicado no cálculo e retornará uma matriz que será analisada com outras matrizes extraídas verificando a probabilidade de isomorfismo.

Palavras-chave: Extração de características, redes complexas, teoria dos grafos, grau dos vértices, matriz de adjacência.

\section{Complex networks in the extraction of features from fish of images}

\begin{abstract}
This paper presents an approach to image analysis using the techniques of Complex Networks for obtaining similarities between images. Initially, the features are extracted from the edge of images, which will be understood computationally as arrays. Through these matrices are generated adjacency matrices, which will be used to check the connections of the vertices (pixels) of images. Extracted from the adjacency matrix will be performed to extract the degree of the vertices to a threshold $T$ and the extraction of the average degree of the vertices. In the extraction of the degree of the vertices each threshold is applied in the calculation and returns an array that will be analyzed with other matrices extracted by checking the likelihood of isomorphism. Keywords: Feature extraction, complex networks, graph theory, degree of vertices, adjacency matrix.
\end{abstract}




\section{Introdução}

Com a ascensão tecnológica nas mais diversas áreas de pesquisa como Medicina e/ou Biologia, torna-se de extrema necessidade a realização mais acelerada de tarefas como identificação de espécies vegetais ou a verificação das características de doenças dos mais variados tipos. Com base nesses problemas, visando maior dinâmica em suas resoluções e também o auxílio aos profissionais do ramo, as pesquisas com Extração de Características de Imagens vêm ganhando espaço na contribuição para um processo mais acelerado e resultados mais exatos utilizando métodos como Transformada de Wavelets, Transformada de Fourier (COSTA; CESAR, 2000), Redes Complexas, entre outros. Neste trabalho, o estudo das Redes Complexas será de extrema importância, pois sua aplicação será essencial na extração das características, em que os resultados obtidos em cada imagem analisada fornecerão uma estrutura de dados que constituirá uma matriz. Cada elemento da matriz representará um pixel da imagem, contendo as cores e os níveis de cinza. A verificação de similaridade será facilitada com a utilização de matrizes e grafos. Cada grafo gerado terá uma quantidade de nós que serão entendidos como os dados das imagens e uma quantidade de arestas que serão interpretadas como as conexões com as regiões das imagens. A comparação dos grafos se dará por meio do isomorfismo, o qual analisa a quantidade de nós, arestas, se o grafo é dirigido ou não, entre outras características. Para se obter um maior número de resultados, a busca por similaridade será realizada em subgrafos por intermédio de métodos estatísticos de reconhecimento de padrões. Se a busca fosse realizada no grafo inteiro, seria necessário que tanto o grafo do banco de dados (já conhecido) quanto o grafo de consulta (conhecido em tempo real) fossem exatamente iguais.

\section{Extração de características de imagens}

As técnicas de análise de imagens e/ou extração de características utilizam as informações extraídas de imagens digitalizadas, tais como informações de cor, textura ou forma como conteúdo a ser compreendido através de uma matriz. Cada elemento da matriz é entendido como um pixel, que corresponde a uma coordenada da imagem FALCÃO et al., 2004). O valor de cada pixel define a intensidade de luz e cor no setor da imagem (SCHWARTZ; PEDRINI, 2003), possibilitando assim, a comparação entre outras imagens visando a obtenção de características similares para a classificação em suas respectivas classes.

Métodos como a utilização do algoritmo genético podem ser utilizados para a extração das características. Os algoritmos genéticos são usados não só para a extração das características, mas também para outros processos de manipulação de imagens como: detecção de borda, segmentação e compressão.

Nas imagens onde consideraremos a característica cor como fator importante na busca de similaridade em imagens, a utilização de histogramas (SCLAROFF et al., 1997) e a métrica Euclidiana são bastante usuais para calcular a distribuição estatística da imagem. Os histogramas são ferramentas muito utilizadas em extração de características. Um exemplo da utilização de histograma (SCLAROFF et al., 1997) é a sua aplicação em uma imagem de exemplo e a comparação com os histogramas das imagens existentes no banco de dados. A comparação entre histogramas pode ser baseada na norma euclidiana (MATHIAS; CONCI, 1997). $\mathrm{Na}$ comparação, devem-se considerar informações baseadas em valores relativos e não absolutos. Isto é importante, pois nem sempre se tem imagens com mesmas dimensões e sim imagens que podem possuir similaridade através de 
conexões em suas subclasses. Diversos estudos relacionados à extração de características de imagens têm utilizado o tema das Redes Complexas no processamento dos dados, utilizando-se dos mesmos para descrever as relações entre os objetos de uma imagem.

\section{Redes Complexas}

Entende-se por rede um conjunto de elementos conectados entre si (NEWMAN, 2003). Tais elementos podem ter relações dos mais variados tipos, onde apenas o estudo dos mesmos seria insuficiente para compreender 0 comportamento de toda a rede. É necessário fazer um levantamento detalhado com as características de cada elemento.

A interpretação da rede fica mais viável quando possui poucos elementos, como por exemplo, uma rede de computadores, onde cada computador tem suas configurações listadas, podendo assim, tirar certas conclusões a respeito da rede. Já quando se trata de uma rede com muito mais elementos, como as características extraídas de uma imagem, devemos considerar um grupo de elementos para poder chegar a uma conclusão e obter um resultado significativo (AIELLO et al., 2000).

Recentemente o estudo das redes começou a analisar o comportamento de redes com milhões de elementos, definindo desta forma, uma rede complexa. Uma rede de grande porte como a rede de seres vivos, depende de vários subgrupos, como o das espécies que, por sua vez, também depende de inúmeros outros subgrupos, para serem formadas, assim como uma imagem dividida em várias partes e/ou subgrupos. Esta classificação se deve ao fato de que estas redes de grande porte, formam estruturas complexas, gerando assim uma imprecisão na sua interpretação (ABERT; BARABÁSI, 1999).
Uma possível solução, para estas indefinições, é uma interação entre o estudo das Redes Complexas com a Teoria dos Grafos.

\section{Teoria dos Grafos}

A Teoria dos Grafos é o ramo da matemática que estuda o conjunto de elementos (nós ou vértices) interligados através de conexões (arestas ou edges), formando assim um grafo. Aplicando no mundo real, um grafo pode representar uma rede social, onde os nós são representados pelas pessoas e a relação entre elas representa as arestas. Um grafo pode também ser aplicado a um sistema de busca, onde deve ser encontrado o menor caminho até seu destino, de modo que a aplicação seja concretizada em tempo reduzido. Um caminho é uma seqüência de nós (A1,A2,A3...An) conectados por arestas $(e 1, e 2),(e 2, e 3), \ldots(e n-1$, en) que serão percorridos até o n-ésimo ponto. Pode ser introduzida na aplicação, uma rotina com laços (loops) (BOAVENTURA NETTO, 2003), que são arestas que iniciam e terminam no mesmo vértice. Grafos podem também ser tratados como árvores, como é o caso de um grafo que representa a árvore genealógica de uma família, em que o nó raiz pode ser o patriarca da família, e gerando "filhos" que são os nós subordinados a ele. Outro exemplo de árvore é a hierarquia de uma imagem dividida em partes, onde a imagem toda seria o pai e as sub-partes seriam os "filhos", "netos" etc.

A representação computacional de um grafo é feita implementando-o com o uso de matrizes, onde os nós são representados como linhas e as arestas como colunas. Existem duas formas de representá-los: com a matriz de adjacência e com a matriz de incidência.

$\mathrm{Na}$ construção de uma matriz de adjacência a conexão entre os nós deve ser analisada. A Figura 1 apresenta um exemplo de 
um grafo simples e sua respectiva matriz de adjacência.

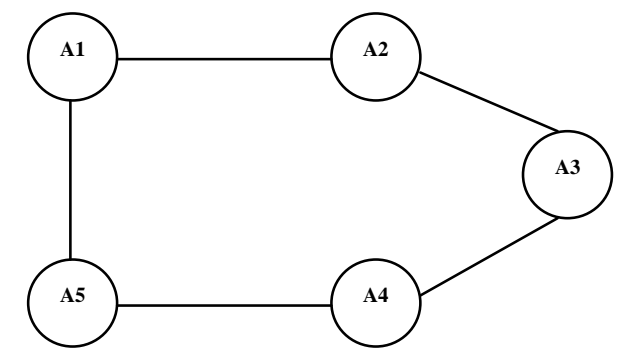

\begin{tabular}{c|ccccc|}
\multicolumn{1}{c}{} & A1 & A2 & A3 & A4 & A5 \\
A1 & 0 & 1 & 0 & 0 & 1 \\
A2 & 1 & 0 & 1 & 0 & 0 \\
A3 & 0 & 1 & 0 & 1 & 0 \\
A4 & 0 & 0 & 1 & 0 & 1 \\
A5 & 1 & 0 & 0 & 1 & 0
\end{tabular}

Figura 1. Grafo simples e sua respectiva matriz de adjacência.

\section{Métodos}

Até o momento os assuntos sobre extração de características de imagens e de redes complexas foram abordados expondo suas principais características detalhadamente, porém os dois temas foram expostos de maneiras distintas sem uma conexão entre eles. Este tópico colocará em destaque a interação entre Extração de Características de imagens e Redes Complexas, de modo que o entendimento do processo de Reconhecimento de Padrões seja facilitado.

Nos experimentos, foi utilizada uma base de dados de contorno de peixes conforme demonstra a Figura 2. O conjunto de imagens utilizadas nos experimentos são disponíveis em (MOKHTARIAN et al., 1996). A ferramenta utilizada para implementação será o Matlab. Para ilustrar o funcionamento dos métodos propostos neste trabalho, utilizaremos a imagem Peixe_2 com uma resolução menor, detalhando todo o processo de extração de características por meio de redes complexas.

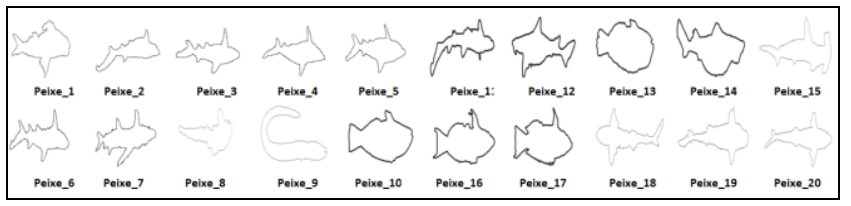

Figura 2. Imagens de peixes.

Para iniciar 0 procedimento, foram extraídas as características das imagens. O processo consiste em extrair as características das bordas das imagens, utilizando o comando EDGE e o atributo canny, que gera uma imagem binária onde 0 valor 1 representa 0 valor preenchido da imagem que é visualizada pela cor preta e o valor 0 correspondente à cor branca. Posteriormente foram armazenadas em um arquivo .txt. A tabela 1 contém as informações do arquivo .txt salvo com as coordenadas $\mathrm{x} e \mathrm{y}$ das bordas da imagem Peixe_2, onde sua resolução foi reduzida em $97 \%$ para exemplificar a metodologia.

Tabela 1. Dados das coordenadas $x$ e $y$ das bodas da imagem salvas no arquivo .txt.

\begin{tabular}{|l|l|}
\hline 53 & 197 \\
\hline 48 & 173 \\
\hline 57 & 143 \\
\hline 43 & 109 \\
\hline 28 & 77 \\
\hline 20 & 45 \\
\hline 27 & 18 \\
\hline 35 & 45 \\
\hline 53 & 76 \\
\hline 80 & 94 \\
\hline 71 & 126 \\
\hline 78 & 155 \\
\hline 81 & 185 \\
\hline 95 & 213 \\
\hline 100 & 247 \\
\hline 101 & 279 \\
\hline 121 & 303 \\
\hline 91 & 318 \\
\hline 84 & 350 \\
\hline 65 & 378 \\
\hline
\end{tabular}




\begin{tabular}{|l|l|}
57 & 351 \\
\hline 53 & 319 \\
\hline 52 & 285 \\
\hline 53 & 253 \\
\hline 53 & 221 \\
\hline
\end{tabular}

As coordenadas $\mathrm{X}, \mathrm{Y}$ dos vértices verificando os valores iguais a 1 referenciando um pixel preenchido de preto. Este processo é essencial, pois como os experimentos foram realizados com base no tema das Redes Complexas, a utilização da borda da imagem é o fator que mais se enquadra no assunto. A borda é uma seqüência de pixels preenchidos, onde os mesmos têm (ou não) conexões com os demais, semelhantes aos nós de um grafo. Esses são os principais pontos que devem ser destacados na Extração de Características de Imagens utilizando Redes Complexas (BACKES et al., 2007).

O processo seguinte deverá obter a distância entre os pontos (vértices) $A\left(x_{1}, y_{1}\right) \mathrm{e}$

$B\left(x_{2}, y_{2}\right)$ que é o comprimento do (módulo) do vetor $|\overrightarrow{A B}|$, isto é, $d(A, B)=|\overrightarrow{A B}|$.

$$
\text { Como }|\overrightarrow{A B}|=B-A=\left(x_{2}-x_{1}, y_{2}-y_{1}\right)
$$

temos a métrica euclidiana. A Equação 1 expõe a fórmula utilizada.

Para isso será contabilizado o número total de vértices da imagem e gerada uma matriz quadrada com a quantidade de linhas e colunas igual ao número de vértices. Adquirida a matriz de distância há a necessidade de normalizá-los. $\mathrm{Na}$ Tabela 2, podemos visualizar os dados da matriz com a distância euclidiana aplicada nos pontos da Tabela 1 de acordo com a Equação 1.

$$
d(A, B)=\sqrt{\left(x_{2}-x_{1}\right)^{2}+\left(y_{2}-y_{1}\right)^{2}}
$$

\section{Equação 1}

\begin{tabular}{|c|c|c|c|c|c|c|c|c|c|c|c|c|c|c|c|c|c|c|c|c|c|c|c|c|}
\hline 0,0 & 4,5 & 4,1 & 88,6 & 122,6 & 55,5 & 80,9 & 153,1 & 121,0 & 106,5 & 73,2 & 48,9 & 30,5 & 44,9 & 68,6 & 95,0 & 125,9 & 126,8 & 156,1 & 181,4 & 154,1 & 122,0 & 88,0 & 56,0 & 24,0 \\
\hline 24,5 & 0,0 & & 64,2 & 8,1 & 1,0 & $\hat{j}, 4$ & 28,7 & 97,1 & 85,2 & & 35,0 & & 1,7 & 0,4 & 8,5 & 49,1 & 151,2 & 80,6 & 5,7 & 78,2 & 46,1 & 112,1 & 0,2 & 48,3 \\
\hline 54,1 & 1,3 & 0,0 & & 72,1 & 04,8 & 28,5 & 00,4 & 67,1 & & & 24,2 & & 79,6 & 112,5 & & 172,3 & 178,3 & 208,8 & 35,1 & 08,0 & 176,0 & 142,1 & 110,1 & 78,1 \\
\hline 88,6 & 64,2 & 36,8 & 0,0 & 35,3 & 68,0 & 924 & 64,5 & 34,5 & 39,9 & 32,8 & 57,8 & 85,0 & 116,3 & 149,3 & 179,6 & 209,1 & 214,4 & 244,5 & 269,9 & 242,4 & 210,2 & 176,2 & 144,3 & 112,4 \\
\hline 122,6 & 98,1 & 72,1 & 35,3 & 0,0 & 33,0 & 9,0 & 32,8 & 25,0 & 54,7 & 65,2 & 92,6 & 120,3 & 151,6 & 184,6 & 214,8 & 244,4 & 249,1 & 278,7 & 303,3 & 275,5 & 243,3 & 209,4 & 177,8 & 146,2 \\
\hline 155,5 & 31,0 & 04,8 & 68,0 & 33,0 & 0,0 & 27,9 & 15,0 & 45,3 & 77,5 & 95,7 & 124,4 & 152,7 & 184,0 & 217,3 & 247,6 & 277,1 & 282,1 & 311,6 & 336,0 & 308,2 & 276,0 & 242,1 & 210,6 & 179,1 \\
\hline 180,9 & 56,4 & 28,5 & 92,4 & 59,0 & 27,9 & 0,0 & 28,2 & 63,6 & 92,7 & 116,6 & 146,2 & 175,5 & 206,5 & 240,4 & 2713 & 300,1 & 306,8 & 336,9 & 362,0 & 334,3 & 302,1 & 268,2 & 236,4 & 204,7 \\
\hline 153,1 & 128,7 & 00,4 & 64,5 & 32,8 & 15,0 & 28,2 & 0,0 & 35,8 & 66,5 & 88,6 & 118,1 & 147,4 & 178,4 & 212,2 & 243,1 & 272,0 & 278,7 & 308,9 & 334,3 & 306,8 & 274,6 & 240,6 & 208,8 & 176,9 \\
\hline 121,0 & 97,1 & 67,1 & 34,5 & 25,0 & 45,3 & 63,6 & 35,8 & 0,0 & 32,4 & 53,1 & 82,9 & 112,5 & 143,3 & 177,3 & 208,6 & 237,0 & 245,0 & 275,7 & 302,2 & 275,0 & 243,0 & 209,0 & 177,0 & 145,0 \\
\hline 106,5 & 85,2 & 54,1 & 39,9 & 54,7 & 77,5 & 92,7 & 66,5 & 32,4 & 0,0 & 33,2 & 61,0 & 91,0 & 119,9 & 154,3 & 186,2 & 213,0 & 224,3 & 256,0 & 284,4 & 258,0 & 226,6 & 193,0 & 161,3 & 129,8 \\
\hline 73,2 & 52,3 & 2 & 32,8 & 65,2 & 95,7 & 6,6 & & & 33,2 & & 29 , & & 90,2 & 124,4 & 155,9 & 183,9 & 193,0 & 224,4 & 252,1 & 25,4 & 193,8 & 160,1 & 128,3 & 96,7 \\
\hline 48,9 & 35,0 & 24,2 & 57,8 & 92,6 & 124,4 & 146,2 & 118,1 & 82,9 & 61,0 & 29,8 & 0,0 & 30,1 & 60,4 & 94,6 & 126,1 & 154,1 & 163,5 & 195,1 & 223,4 & 197,1 & 165,9 & 132,6 & 101,1 & 70,6 \\
\hline 30,5 & 35,1 & 48,4 & 85,0 & 120,3 & 152,7 & 175,5 & 147,4 & 112,5 & 91,0 & 59,8 & 30,1 & 0,0 & 31,3 & 64,8 & 96,1 & 124,6 & 133,4 & 165,0 & 193,7 & 167,7 & 136,9 & 104,1 & 73,5 & 45,6 \\
\hline 44,9 & 61,7 &, 6 & 6,3 & 51,6 & 184,0 & 206,5 & 178,4 & 143,3 & 119,9 & 90, & 60,4 & 31, & 0,0 & 34,4 & 66 & 93, & 105,1 & 137,4 & 167,7 & 143,1 & 114,0 & 33,9 & 58,0 & 42,8 \\
\hline 68,6 & D,4 & 2,5 & 49,3 & 184,6 & 217,3 & 240,4 & 212,2 & 177,3 & 154,3 & 124,4 & 94,6 & 64,8 & 34,4 & 0 & 320 & 50 & 71,6 & 104,2 & 135,6 & 112,5 & 86,0 & 51,2 & 47,4 & 53,7 \\
\hline 95,0 & 118,5 & 142,9 & 179,6 & 214,8 & 247,6 & 271,3 & 243,1 & 208,6 & 186,2 & 155,9 & 126,1 & 96,1 & 66,3 & 32,0 & 0,0 & 31,2 & 40,3 & 73,0 & 105,3 & 84,4 & 62,5 & 49,4 & 54,6 & 75,3 \\
\hline 125,9 & 149,1 & 172,3 & 209,1 & 244,4 & 277,1 & 300,1 & 272,0 & 237,0 & 213,0 & 183,9 & 154,1 & 124,6 & 93,7 & 59,8 & 31,2 & 0,0 & 33,5 & 59,8 & 93,6 & 80,0 & 69,9 & 71,3 & 84,4 & 106,5 \\
\hline 126,8 & 151,2 & 178,3 & 214,4 & 49,1 & 282,1 & 306,8 & 278,7 & 245,0 & 224,3 & 193,0 & 163,5 & 133,4 & 105,1 & & & & & $0<, 0$ & 0,7 & $4 l, 4$ & 38,0 & & 75,3 & 104,2 \\
\hline 156,1 & 180,6 & 08,8 & 244,5 & 278,7 & 311,6 & 336,9 & 308,9 & 275,7 & 256,0 & 224,4 & 195,1 & 165,0 & 137,4 & 104,2 & 73,0 & 59,8 & 32,8 & 0,0 & 33,8 & 27,0 & 43,8 & 72,4 & 101,8 & 132,7 \\
\hline 181,4 & 205,7 & 235,1 & 269,9 & 303,3 & 336,0 & 362,0 & 334,3 & 302,2 & 284,4 & 252,1 & 223,4 & 193,7 & 167,7 & 135,6 & 105,3 & 93,6 & 65,4 & 33,8 & 0,0 & 28,2 & 60,2 & 93,9 & 125,6 & 157,5 \\
\hline 154,1 & 178,2 & 208,0 & 242,4 & 275,5 & 308,2 & 334,3 & 306,8 & 275,0 & 258,0 & 225,4 & 197,1 & 167,7 & 143,1 & 112,5 & 84,4 & 80,0 & 47,4 & 27,0 & 28,2 & 0,0 & 32,2 & 66,2 & 98,1 & 130,1 \\
\hline 122,0 & 146,1 & 176,0 & 210,2 & 243,3 & 276,0 & 302,1 & 274,6 & 243,0 & 226,6 & 193,8 & 165,9 & 136,9 & 114,0 & 86,0 & 62,5 & 69,9 & 38,0 & 43,8 & 60,2 & 32,2 & 0,0 & 34,0 & 66,0 & 98,0 \\
\hline 88,0 & 112,1 & 142,1 & 176,2 & 209,4 & 242,1 & 268,2 & 240,6 & 209,0 & 193,0 & 160,1 & 132,6 & 104,1 & 83,9 & 61,2 & 49,4 & 71,3 & 51,1 & 72,4 & 93,9 & 66,2 & 34,0 & 0,0 & 32,0 & 64,0 \\
\hline 56,0 & 80,2 & 110,1 & 144,3 & 177,8 & 210,6 & 236,4 & 208,8 & 177,0 & 161,3 & 128,3 & 101,1 & 73,5 & 58,0 & 47,4 & 54,6 & 84,4 & 75,3 & 101,8 & 125,6 & 98,1 & 66,0 & 32,0 & 0,0 & 32,0 \\
\hline 24,0 & 48,3 & 78,1 & 112,4 & 146,2 & 179,1 & 204,7 & 176,9 & 145,0 & 129,8 & 96,7 & 70,6 & 45,6 & 42,8 & 53,7 & 75,3 & 106,5 & 104,2 & 132,7 & 157,5 & 130,1 & 98,0 & 64,0 & 32,0 & 0,0 \\
\hline
\end{tabular}

Tabela 2. Matriz gerada com a aplicação do método da distância euclidiana . 
A normalização dos dados consistirá em adaptar valores extremamente altos (ou baixos) e "transformá-los" em valores que estarão dentro de uma faixa de valores padrão. Em outras palavras adaptará valores que vão de 20 a 35000 no intervalo que vai de 0 a 1 . Esse processo de normalização é demonstrado na Tabela 3.
Tabela 3. Matriz de Distâncias com os dados normalizados.

\begin{tabular}{|c|c|c|c|c|c|c|c|c|c|c|c|c|c|c|c|c|c|c|c|c|c|c|c|c|}
\hline 0,00 & 0,07 & 0,15 & 0,24 & 0,34 & 0,43 & 0,50 & 0,42 & 0,33 & 0,29 & 0,20 & 0,14 & 0,08 & 0,12 & 0,19 & 0,26 & 0,35 & 0,35 & 0,43 & 0,50 & 0,43 & 0,34 & 0,24 & 0,15 & 0,07 \\
\hline 0,07 & 0,00 & 0,09 & 0,18 & 0,27 & 0,36 & 0,43 & 0,36 & 0,27 & 0,24 & 0,14 & 0,10 & 0,10 & 0,17 & 0,25 & 0,33 & 0,41 & 0,42 & 0,50 & 0,57 & 0,49 & 0,40 & 0,31 & 0,22 & 0,13 \\
\hline 0,15 & 0,09 & 0,00 & 0,10 & 0,20 & 0,29 & 0,36 & 0,28 & 0,19 & 0,15 & 0,06 & 0,07 & 0,13 & 0,22 & 0,31 & 0,39 & 0,48 & 0,49 & 0,58 & 0,65 & 0,57 & 0,49 & 0,39 & 0,30 & 0,22 \\
\hline 0,24 & 0,18 & 0,10 & 0,00 & 0,10 & 0,19 & 0,26 & 0,18 & 0,10 & 0,11 & 0,09 & 0,16 & 0,23 & 0,32 & 0,41 & 0,50 & 0,58 & 0,59 & 0,68 & 0,75 & 0,67 & 0,58 & 0,49 & 0,40 & 0,31 \\
\hline 0,34 & 0,27 & 0,20 & 0,10 & 0,00 & 0,09 & 0,16 & 0,09 & 0,07 & 0,15 & 0,18 & 0,26 & 0,33 & 0,42 & 0,51 & 0,59 & 0,68 & 0,69 & 0,77 & 0,84 & 0,76 & 0,67 & 0,58 & 0,49 & 0,40 \\
\hline 0,43 & 0,36 & 0,29 & 0,19 & 0,09 & 0,00 & 0,08 & 0,04 & 0,13 & 0,21 & 0,26 & 0,34 & 0,42 & 0,51 & 0,60 & 0,68 & 0,77 & 0,78 & 0,86 & 0,93 & 0,85 & 0,76 & 0,67 & 0,58 & 0,49 \\
\hline 0,50 & 0,43 & 0,36 & 0,26 & 0,16 & 0,08 & 0,00 & 0,08 & 0,18 & 0,26 & 0,32 & 0,40 & 0,48 & 0,57 & 0,66 & 0,75 & 0,83 & 0,85 & 0,93 & 1,00 & 0,92 & 0,83 & 0,74 & 0,65 & 0,57 \\
\hline 0,42 & 0,36 & 0,28 & 0,18 & 0,09 & 0,04 & 0,08 & 0,00 & 0,10 & 0,18 & 0,24 & 0,33 & 0,41 & 0,49 & 0,59 & 0,67 & 0,75 & 0,77 & 0,85 & 0,92 & 0,85 & 0,76 & 0,66 & 0,58 & 0,49 \\
\hline 0,33 & 0,27 & 0,19 & 0,10 & 0,07 & 0,13 & 0,18 & 0,10 & 0,00 & 0,09 & 0,15 & 0,23 & 0,31 & 0,40 & 0,49 & 0,58 & 0,65 & 0,68 & 0,76 & 0,83 & 0,76 & 0,67 & 0,58 & 0,49 & 0,40 \\
\hline 0,29 & 0,24 & 0,15 & 0,11 & 0,15 & 0,21 & 0,26 & 0,18 & 0,09 & 0,00 & 0,09 & 0,17 & 0,25 & 0,33 & 0,43 & 0,51 & 0,59 & 0,62 & 0,71 & 0,79 & 0,71 & 0,63 & 0,53 & 0,45 & 0,36 \\
\hline 0,20 & 0,14 & 0,06 & 0,09 & 0,18 & 0,26 & 0,32 & 0,24 & 0,15 & 0,09 & 0,00 & 0,08 & 0,17 & 0,25 & 0,34 & 0,43 & 0,51 & 0,53 & 0,62 & 0,70 & 0,62 & 0,54 & 0,44 & 0,35 & 0,27 \\
\hline 0,14 & 0,10 & 0,07 & 0,16 & 0,26 & 0,34 & 0,40 & 0,33 & 0,23 & 0,17 & 0,08 & 0,00 & 0,08 & 0,17 & 0,26 & 0,35 & 0,43 & 0,45 & 0,54 & 0,62 & 0,54 & 0,46 & 0,37 & 0,28 & 0,19 \\
\hline 0,08 & 0,10 & 0,13 & 0,23 & 0,33 & 0,42 & 0,48 & 0,41 & 0,31 & 0,25 & 0,17 & 0,08 & 0,00 & 0,09 & 0,18 & 0,27 & 0,34 & 0,37 & 0,46 & 0,53 & 0,46 & 0,38 & 0,29 & 0,20 & 0,13 \\
\hline 0,12 & 0,17 & 0,22 & 0,32 & 0,42 & 0,51 & 0,57 & 0,49 & 0,40 & 0,33 & 0,25 & 0,17 & 0,09 & 0,00 & 0,09 & 0,18 & 0,26 & 0,29 & 0,38 & 0,46 & 0,40 & 0,31 & 0,23 & 0,16 & 0,12 \\
\hline 0,19 & 0,25 & 0,31 & 0,41 & 0,51 & 0,60 & 0,66 & 0,59 & 0,49 & 0,43 & 0,34 & 0,26 & 0,18 & 0,09 & 0,00 & 0,09 & 0,17 & 0,20 & 0,29 & 0,37 & 0,31 & 0,24 & 0,17 & 0,13 & 0,15 \\
\hline 0,26 & 0,33 & 0,39 & 0,50 & 0,59 & 0,68 & 0,75 & 0,67 & 0,58 & 0,51 & 0,43 & 0,35 & 0,27 & 0,18 & 0,09 & 0,00 & 0,09 & 0,11 & 0,20 & 0,29 & 0,23 & 0,17 & 0,14 & 0,15 & 0,21 \\
\hline 0,35 & 0,41 & 0,48 & 0,58 & 0,68 & 0,77 & 0,83 & 0,75 & 0,65 & 0,59 & 0,51 & 0,43 & 0,34 & 0,26 & 0,17 & 0,09 & 0,00 & 0,09 & 0,17 & 0,26 & 0,22 & 0,19 & 0,20 & 0,23 & 0,29 \\
\hline 0,35 & 0,42 & 0,49 & 0,59 & 0,69 & 0,78 & 0,85 & 0,77 & 0,68 & 0,62 & 0,53 & 0,45 & 0,37 & 0,29 & 0,20 & 0,11 & 0,09 & 0,00 & 0,09 & 0,18 & 0,13 & 0,11 & 0,14 & 0,21 & 0,29 \\
\hline 0,43 & 0,50 & 0,58 & 0,68 & 0,77 & 0,86 & 0,93 & 0,85 & 0,76 & 0,71 & 0,62 & 0,54 & 0,46 & 0,38 & 0,29 & 0,20 & 0,17 & 0,09 & 0,00 & 0,09 & 0,07 & 0,12 & 0,20 & 0,28 & 0,37 \\
\hline 0,50 & 0,57 & 0,65 & 0,75 & 0,84 & 0,93 & 1,00 & 0,92 & 0,83 & 0,79 & 0,70 & 0,62 & 0,53 & 0,46 & 0,37 & 0,29 & 0,26 & 0,18 & 0,09 & 0,00 & 0,08 & 0,17 & 0,26 & 0,35 & 0,43 \\
\hline 0,43 & 0,49 & 0,57 & 0,67 & 0,76 & 0,85 & 0,92 & 0,85 & 0,76 & 0,71 & 0,62 & 0,54 & 0,46 & 0,40 & 0,31 & 0,23 & 0,22 & 0,13 & 0,07 & 0,08 & 0,00 & 0,09 & 0,18 & 0,27 & 0,36 \\
\hline 0,34 & 0,40 & 0,49 & 0,58 & 0,67 & 0,76 & 0,83 & 0,76 & 0,67 & 0,63 & 0,54 & 0,46 & 0,38 & 0,31 & 0,24 & 0,17 & 0,19 & 0,11 & 0,12 & 0,17 & 0,09 & 0,00 & 0,09 & 0,18 & 0,27 \\
\hline 0,24 & 0,31 & 0,39 & 0,49 & 0,58 & 0,67 & 0,74 & 0,66 & 0,58 & 0,53 & 0,44 & 0,37 & 0,29 & 0,23 & 0,17 & 0,14 & 0,20 & 0,14 & 0,20 & 0,26 & 0,18 & 0,09 & 0,00 & 0,09 & 0,18 \\
\hline 0,15 & 0,22 & 0,30 & 0,40 & 0,49 & 0,58 & 0,65 & 0,58 & 0,49 & 0,45 & 0,35 & 0,28 & 0,20 & 0,16 & 0,13 & 0,15 & 0,23 & 0,21 & 0,28 & 0,35 & 0,27 & 0,18 & 0,09 & 0,00 & 0,09 \\
\hline 0,07 & 0,13 & 0,22 & 0,31 & 0,40 & 0,49 & 0,57 & 0,49 & 0,40 & 0,36 & 0,27 & 0,19 & 0,13 & 0,12 & 0,15 & 0,21 & 0,29 & 0,29 & 0,37 & 0,43 & 0,36 & 0,27 & 0,18 & 0,09 & 0,00 \\
\hline
\end{tabular}

Uma vez que os dados estejam normalizados, o próximo item da sequência será gerar a matriz de adjacência contendo também o número de colunas e o número de linhas com o mesmo valor do número de vértices contabilizados pela extração da borda. Através desta matriz de adjacência foram computadas todas as conexões de cada vértice com base em limiares preestabelecidos, onde o número de conexões de cada vértice determina o grau do mesmo. Os limiares preestabelecidos foram 0.1 (Tabela 4), 0.25 (Tabela 5), 0.5 (Tabela 6) , 0.75 (Tabela 7) e 0.95 (Tabela 8). Quanto maior 0 limiar, maior será o grau de cada vértice, definindo assim um resultado mais preciso, porém é importante que haja uma variação dos valores dos limiares para que seja realizada uma análise mais concreta da similaridade. Com os graus dos vértices registrados, será necessário encontrar o grau máximo e o grau mínimo dos vértices para cada limiar aplicado, com o resultado obtido deverá ser encontrado também o grau médio, onde deverá ser somado o valor do grau máximo e o valor do grau mínimo dividido por dois. 
Tabela 4. Matriz de Adjacência gerada pelo limiar 0.1 e seus respectivos Graus.

\begin{tabular}{|c|c|c|c|c|c|c|c|c|c|c|c|c|c|c|c|c|c|c|c|c|c|c|c|c|}
\hline & & 0 & 0 & & & & & & & & & & & & & & & & & & & & & \\
\hline & & 1 & & & & & & & & & & & & & & & & 0 & 0 & & O & 0 & & 0 \\
\hline & & 0 & & & & & & & & & & & & & & & & & C & & & & & \\
\hline & & 0 & & & & & & & & & & & & & & & & & 0 & & & 0 & & D \\
\hline & 0 & 0 & & & & & & & & & & 0 & & & & & & & 0 & 0 & 0 & 0 & & 0 \\
\hline & & 0 & & & & & & & & & & & & & & & & 0 & 0 & & & 0 & & \\
\hline & & 0 & & & & & & & & & & 0 & & & & & 0 & 0 & 0 & 0 & 0 & 0 & 0 & \\
\hline & 0 & 0 & & & & & & & & & & 0 & & & & & 0 & & 0 & 0 & 0 & 0 & 0 & 0 \\
\hline & 0 & 0 & & & & & & & & & & 0 & & & 0 & 0 & 0 & 0 & 0 & 0 & 0 & 0 & 0 & 10 \\
\hline & 0 & 0 & & & & & & & & & 0 & 0 & & & & & 0 & 0 & 0 & 0 & 0 & 0 & 0 & 0 \\
\hline & 0 & 1 & & & & & & & & & 1 & 0 & & & & 0 & 0 & 0 & 0 & 0 & 0 & 0 & 0 & 0 \\
\hline & 1 & 1 & & & & & & & & & & & & & & & 0 & 0 & 0 & 0 & 0 & 0 & & I \\
\hline & 1 & 0 & & & & & & & O & & 1 & 0 & & & & & 0 & 0 & 0 & 0 & 0 & 0 & 0 & 0 \\
\hline & 0 & 0 & & & & & & & & & & & & & & & & & 0 & 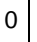 & 0 & & & 0 \\
\hline & 0 & 0 & & & & & & & 0 & 0 & 0 & 0 & & & & & o & 0 & 0 & 0 & 0 & 0 & & 0 \\
\hline & 0 & 0 & & & & & & & & & & 0 & & & 0 & & 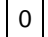 & 0 & 0 & 0 & 0 & 0 & 0 & 0 \\
\hline & 0 & 0 & & & & & & & & & & & & & & & & & & & & & & \\
\hline & 0 & 0 & & & & & & 0 & 0 & 0 & 0 & 0 & 0 & 0 & 0 & 1 & 0 & 1 & 0 & 0 & 0 & 0 & 0 & 0 \\
\hline & 0 & 0 & & & & & & & & & & 10 & & & & & & & 1 & 1 & 0 & 0 & 0 & \\
\hline & 0 & 0 & & & & & & & & 0 & 0 & 0 & & & 0 & 10 & 0 & 1 & 0 & 1 & 0 & 0 & 0 & 0 \\
\hline & 0 & 0 & & & & & & & 0 & & 0 & 0 & & & 0 & 0 & 0 & & 1 & 0 & 1 & 0 & 0 & 0 \\
\hline & 0 & 0 & 0 & 0 & & 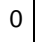 & & & 0 & 10 & 0 & 0 & 10 & 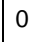 & 0 & 0 & 0 & 0 & 0 & 1 & 0 & 1 & 0 & 0 \\
\hline & 0 & 0 & & & & & & 0 & 0 & 0 & 0 & 0 & 0 & 0 & 0 & 0 & 0 & 1 & 0 & 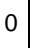 & 7 & 0 & 1 & 0 \\
\hline & 0 & 0 & 0 & 0 & 0 & 0 & & 0 & 0 & 0 & 0 & 0 & 0 & 10 & 0 & c & 0 & 0 & 0 & 0 & 0 & 1 & 0 & \\
\hline & & & & & & & & & & & & & & & & & & & & & & & & \\
\hline
\end{tabular}

Grau Máximo

Grau Mínimo

Grau Médio

$$
4
$$

Tabela 5. Matriz de Adjacência gerada pelo limiar 0.25 e seus respectivos Graus. 


\begin{tabular}{|c|c|c|c|c|c|c|c|c|c|c|c|c|c|c|c|c|c|c|c|c|c|c|c|c|}
\hline 0 & 1 & 1 & 1 & 0 & 0 & 0 & 0 & 0 & 0 & 1 & 1 & 1 & 1 & 1 & 0 & 0 & 0 & 0 & 0 & 0 & 0 & 1 & 1 & 1 \\
\hline 1 & 0 & 1 & 1 & 0 & 0 & 0 & 0 & 0 & 1 & 1 & 1 & 1 & 1 & 1 & 0 & 0 & 0 & 0 & 0 & 0 & 0 & 0 & 1 & 1 \\
\hline 1 & 1 & 0 & 1 & 1 & 0 & 0 & 0 & 1 & 1 & 1 & 1 & 1 & 1 & 0 & 0 & 0 & 0 & 0 & 0 & 0 & 0 & 0 & 0 & 1 \\
\hline 1 & 1 & 1 & 0 & 1 & 1 & 0 & 1 & 1 & 1 & 1 & 1 & 1 & 0 & 0 & 0 & 0 & 0 & 0 & 0 & 0 & 0 & 0 & 0 & 0 \\
\hline 0 & 0 & 1 & 1 & 0 & 1 & 1 & 1 & 1 & 1 & 1 & 0 & 0 & 0 & 0 & 0 & 0 & 0 & 0 & 0 & 0 & 0 & 0 & 0 & 0 \\
\hline 0 & 0 & 0 & 1 & 1 & 0 & 1 & 1 & 1 & 1 & 0 & 0 & 0 & 0 & 0 & 0 & 0 & 0 & 0 & 0 & 0 & 0 & 0 & 0 & 0 \\
\hline 0 & 0 & 0 & 0 & 1 & 1 & 0 & 1 & 1 & 0 & 0 & 0 & 0 & 0 & 0 & 0 & 0 & 0 & 0 & 0 & 0 & 0 & 0 & 0 & 0 \\
\hline 0 & 0 & 0 & 1 & 1 & 1 & 1 & 0 & 1 & 1 & 1 & 0 & 0 & 0 & 0 & 0 & 0 & 0 & 0 & 0 & 0 & 0 & 0 & 0 & 0 \\
\hline 0 & 0 & 1 & 1 & 1 & 1 & 1 & 1 & 0 & 1 & 1 & 1 & 0 & 0 & 0 & 0 & 0 & 0 & 0 & 0 & 0 & 0 & 0 & 0 & 0 \\
\hline 0 & 1 & 1 & 1 & 1 & 1 & 0 & 1 & 1 & 0 & 1 & 1 & 0 & 0 & 0 & 0 & 0 & 0 & 0 & 0 & 0 & 0 & 0 & 0 & 0 \\
\hline 1 & 1 & 1 & 1 & 1 & 0 & 0 & 1 & 1 & 1 & 0 & 1 & 1 & 1 & 0 & 0 & 0 & 0 & 0 & 0 & 0 & 0 & 0 & 0 & 0 \\
\hline 1 & 1 & 1 & 1 & 0 & 0 & 0 & 0 & 1 & 1 & 1 & 0 & 1 & 1 & 0 & 0 & 0 & 0 & 0 & 0 & 0 & 0 & 0 & 0 & 1 \\
\hline 1 & 1 & 1 & 1 & 0 & 0 & 0 & 0 & 0 & 0 & 1 & 1 & 0 & 1 & 1 & 0 & 0 & 0 & 0 & 0 & 0 & 0 & 0 & 1 & 1 \\
\hline 1 & 1 & 1 & 0 & 0 & 0 & 0 & 0 & 0 & 0 & 1 & 1 & 1 & 0 & 1 & 1 & 0 & 0 & 0 & 0 & 0 & 0 & 1 & 1 & 1 \\
\hline 1 & 1 & 0 & 0 & 0 & 0 & 0 & 0 & 0 & 0 & 0 & 0 & 1 & 1 & 0 & 1 & 1 & 1 & 0 & 0 & 0 & 1 & 1 & 1 & 1 \\
\hline 0 & 0 & 0 & 0 & 0 & 0 & 0 & 0 & 0 & 0 & 0 & 0 & 0 & 1 & 1 & 0 & 1 & 1 & 1 & 0 & 1 & 1 & 1 & 1 & 1 \\
\hline 0 & 0 & 0 & 0 & 0 & 0 & 0 & 0 & 0 & 0 & 0 & 0 & 0 & 0 & 1 & 1 & 0 & 1 & 1 & 0 & 1 & 1 & 1 & 1 & 0 \\
\hline 0 & 0 & 0 & 0 & 0 & 0 & 0 & 0 & 0 & 0 & 0 & 0 & 0 & 0 & 1 & 1 & 1 & 0 & 1 & 1 & 1 & 1 & 1 & 1 & 0 \\
\hline 0 & 0 & 0 & 0 & 0 & 0 & 0 & 0 & 0 & 0 & 0 & 0 & 0 & 0 & 0 & 1 & 1 & 1 & 0 & 1 & 1 & 1 & 1 & 0 & 0 \\
\hline 0 & 0 & 0 & 0 & 0 & 0 & 0 & 0 & 0 & 0 & 0 & 0 & 0 & 0 & 0 & 0 & 0 & 1 & 1 & 0 & 1 & 1 & 0 & 0 & 0 \\
\hline 0 & 0 & 0 & 0 & 0 & 0 & 0 & 0 & 0 & 0 & 0 & 0 & 0 & 0 & 0 & 1 & 1 & 1 & 1 & 1 & 0 & 1 & 1 & 0 & 0 \\
\hline 0 & 0 & 0 & 0 & 0 & 0 & 0 & 0 & 0 & 0 & 0 & 0 & 0 & 0 & 1 & 1 & 1 & 1 & 1 & 1 & 1 & 0 & 1 & 1 & 0 \\
\hline 1 & 0 & 0 & 0 & 0 & 0 & 0 & 0 & 0 & 0 & 0 & 0 & 0 & 1 & 1 & 1 & 1 & 1 & 1 & 0 & 1 & 1 & 0 & 1 & 1 \\
\hline 1 & 1 & 0 & 0 & 0 & 0 & 0 & 0 & 0 & 0 & 0 & 0 & 1 & 1 & 1 & 1 & 1 & 1 & 0 & 0 & 0 & 1 & 1 & 0 & 1 \\
\hline 1 & 1 & 1 & 0 & 0 & 0 & 0 & 0 & 0 & 0 & 0 & 1 & 1 & 1 & 1 & 1 & 0 & 0 & 0 & 0 & 0 & 0 & 1 & 1 & 0 \\
\hline
\end{tabular}

\begin{tabular}{|c|c|c|}
\hline Grau Máximo & Grau Mínimo & Grau Médio \\
\hline 11 & 4 & 7,5 \\
\hline
\end{tabular}


Tabela 6. Matriz de Adjacência gerada pelo limiar 0.5 e seus respectivos Graus.

\begin{tabular}{|c|c|c|c|c|c|c|c|c|c|c|c|c|c|c|c|c|c|c|c|c|c|c|}
\hline & 1 & 1 & 1 & 1 & 1 & 1 & 1 & 1 & 1 & 1 & 1 & 1 & \begin{tabular}{|l|l|l}
1 & 1
\end{tabular} & \begin{tabular}{l|l}
1 & 1
\end{tabular} & 1 & 1 & 1 & 0 & \begin{tabular}{l|l}
1 & 1
\end{tabular} & \begin{tabular}{l|l|l|}
1 & 1
\end{tabular} & 1 & \\
\hline & 0 & 1 & 1 & & 1 & 1 & 1 & 1 & & 1 & 1 & 1 & 1 & \begin{tabular}{l|l}
1 & 1
\end{tabular} & 1 & 1 & 1 & 0 & \begin{tabular}{l|l}
1 & 1
\end{tabular} & \begin{tabular}{l|l}
1 & 1
\end{tabular} & & 1 \\
\hline & & 0 & 1 & & & 1 & 1 & 1 & & & 1 & 1 & 1 & \begin{tabular}{l|l}
1 & 1
\end{tabular} & 1 & 1 & 0 & 0 & \begin{tabular}{l|l}
0 & 1
\end{tabular} & 1 & 1 & 1 \\
\hline & & 1 & 0 & & & 1 & & & & & 1 & 1 & \begin{tabular}{|l|l}
1 & 1
\end{tabular} & \begin{tabular}{l|l}
1 & 1
\end{tabular} & 0 & 0 & 0 & 0 & \begin{tabular}{l|l}
0 & 0
\end{tabular} & ) & 1 & 1 \\
\hline 1 & 1 & 1 & 1 & 0 & & 1 & 1 & 1 & & 1 & 1 & 1 & 0 & \begin{tabular}{l|l}
0 & 0
\end{tabular} & 0 & 0 & 0 & 0 & \begin{tabular}{l|l}
0 & 0
\end{tabular} & 0 & 1 & 1 \\
\hline 1 & 1 & 1 & 1 & 1 & 0 & 1 & 1 & & 1 & 1 & 1 & 1 & 0 & \begin{tabular}{ll|l}
0 & 0
\end{tabular} & 0 & 0 & 0 & 0 & \begin{tabular}{l|l}
0 & 0
\end{tabular} & 0 & 0 & 1 \\
\hline 1 & 1 & 1 & 1 & 1 & 1 & 0 & 1 & 1 & 1 & 1 & 1 & 1 & \begin{tabular}{|l|l|l|l|}
0 & 0
\end{tabular} & \begin{tabular}{l|l}
0 & 0
\end{tabular} & 0 & 0 & 0 & 0 & \begin{tabular}{l|l}
0 & 0
\end{tabular} & 0 & 0 & 0 \\
\hline 1 & 1 & 1 & 1 & 1 & 1 & 1 & 0 & 1 & 1 & 1 & 1 & 1 & 0 & 0 & 0 & 0 & 0 & 0 & 0 & 0 & 0 & 1 \\
\hline 1 & 1 & 1 & 1 & 1 & 1 & 1 & 1 & 1 & 1 & 1 & 1 & 1 & \begin{tabular}{|l|l|l} 
& 1
\end{tabular} & \begin{tabular}{l|l}
1 & 0
\end{tabular} & 0 & 0 & 0 & 0 & \begin{tabular}{l|l}
0 & 0
\end{tabular} & 0 & 1 & 1 \\
\hline 1 & 1 & 1 & 1 & 1 & 1 & 1 & 1 & 1 & 0 & 1 & 1 & 1 & 1 & 0 & 0 & 0 & 0 & 0 & 0 & 0 & 1 & 1 \\
\hline 1 & 1 & 1 & 1 & 1 & 1 & 1 & 1 & 1 & 1 & 0 & 1 & 1 & 1 & \begin{tabular}{l|l}
1 & 1
\end{tabular} & 0 & 0 & 0 & 0 & \begin{tabular}{l|l}
0 & 0
\end{tabular} & 1 & 1 & 1 \\
\hline 1 & 1 & 1 & 1 & 1 & 1 & 1 & 1 & 1 & 1 & 1 & 0 & 1 & 1 & \begin{tabular}{l|l}
1 & 1
\end{tabular} & 1 & 1 & 0 & 0 & \begin{tabular}{l|l}
0 & 1
\end{tabular} & 1 & 1 & 1 \\
\hline 1 & 1 & 1 & 1 & 1 & 1 & 1 & 1 & 1 & 1 & 1 & 1 & 0 & 1 & 1 & 1 & 1 & 1 & 0 & \begin{tabular}{l|l}
1 & 1
\end{tabular} & \begin{tabular}{l|l}
1 & 1
\end{tabular} & 1 & 1 \\
\hline 1 & 1 & 1 & 1 & 1 & 0 & 0 & 1 & 1 & & 1 & 1 & 1 & 0 & \begin{tabular}{l|l}
1 & 1
\end{tabular} & 1 & 1 & 1 & 1 & \begin{tabular}{l|l}
1 & 1
\end{tabular} & 1 & 1 & 1 \\
\hline 1 & 1 & 1 & 1 & & 0 & 0 & 0 & & 1 & 1 & 1 & 1 & 0 & 1 & 1 & 1 & 1 & 1 & \begin{tabular}{l|l}
1 & 1
\end{tabular} & \begin{tabular}{l|l}
1 & 1
\end{tabular} & 1 & 1 \\
\hline 1 & 1 & 1 & 1 & 0 & 0 & 0 & 0 & 0 & 0 & 1 & 1 & 1 & 1 & \begin{tabular}{l|l}
1 & 0
\end{tabular} & 1 & 1 & 1 & 1 & \begin{tabular}{l|l}
1 & 1
\end{tabular} & \begin{tabular}{l|l|}
1 & 1
\end{tabular} & 1 & 1 \\
\hline 1 & 1 & 1 & 0 & & O & 0 & 0 & & 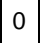 & 0 & 1 & 1 & 1 & 1 & 0 & 1 & 1 & 1 & \begin{tabular}{l|l}
1 & 1
\end{tabular} & \begin{tabular}{l|l}
1 & 1
\end{tabular} & 1 & 1 \\
\hline 1 & 1 & 1 & 0 & 0 & 0 & 0 & 0 & 0 & 0 & 0 & 1 & 1 & 1 & \begin{tabular}{l|l}
1 & 1
\end{tabular} & 1 & 0 & 1 & 1 & \begin{tabular}{l|l}
1 & 1
\end{tabular} & \begin{tabular}{l|l}
1 & 1
\end{tabular} & 1 & 1 \\
\hline 1 & 1 & 0 & 0 & 0 & 0 & 0 & 0 & 0 & 0 & 0 & 0 & 1 & 1 & \begin{tabular}{l|l}
1 & 1
\end{tabular} & 1 & 1 & 0 & 1 & \begin{tabular}{l|l}
1 & 1
\end{tabular} & \begin{tabular}{l|l}
1 & 1
\end{tabular} & 1 & 1 \\
\hline 0 & 0 & 0 & 0 & 0 & 0 & 0 & 0 & 0 & 0 & 0 & 0 & 0 & 1 & \begin{tabular}{l|l}
1 & 1
\end{tabular} & 1 & 1 & 1 & 0 & \begin{tabular}{l|l}
1 & 1
\end{tabular} & \begin{tabular}{l|l}
1 & 1
\end{tabular} & 1 & 1 \\
\hline 1 & 1 & 0 & 0 & 0 & 0 & 0 & 0 & 0 & 0 & 0 & 0 & 1 & \begin{tabular}{|l|l}
1 & 1
\end{tabular} & \begin{tabular}{l|l}
1 & 1
\end{tabular} & 1 & 1 & 1 & 1 & \begin{tabular}{l|l}
0 & 1
\end{tabular} & $\mid$\begin{tabular}{l|l}
1 & 1
\end{tabular} & 1 & 1 \\
\hline 1 & 1 & 1 & 0 & 0 & 0 & 0 & 0 & 0 & 0 & 0 & 1 & 1 & 1 & \begin{tabular}{l|l}
1 & 1
\end{tabular} & 1 & 1 & 1 & 1 & \begin{tabular}{l|l}
1 & 0
\end{tabular} & 1 & 1 & 1 \\
\hline 1 & 1 & 1 & 1 & 0 & 0 & 0 & 0 & 0 & 0 & 1 & 1 & 1 & \begin{tabular}{|l|l}
1 & 1
\end{tabular} & \begin{tabular}{l|l|}
1 & 1
\end{tabular} & 1 & 1 & 1 & 1 & \begin{tabular}{l|l}
1 & 1
\end{tabular} & \begin{tabular}{l|l}
1 & 0
\end{tabular} & 1 & 1 \\
\hline 1 & 1 & 1 & 1 & 1 & 0 & 0 & 0 & 1 & 1 & 1 & 1 & 1 & 1 & \begin{tabular}{l|l}
1 & 1
\end{tabular} & 1 & 1 & 1 & 1 & \begin{tabular}{l|l}
1 & 1
\end{tabular} & \begin{tabular}{l|l}
1 & 1
\end{tabular} & 0 & 1 \\
\hline 1 & 1 & 1 & 1 & 1 & 1 & 0 & 1 & 1 & 1 & 1 & 1 & 1 & \begin{tabular}{|l|l|} 
& 1
\end{tabular} & \begin{tabular}{l|l}
1 & 1
\end{tabular} & 1 & 1 & 1 & 1 & \begin{tabular}{l|l}
1 & 1
\end{tabular} & \begin{tabular}{l|l}
1 & 1
\end{tabular} & 1 & 0 \\
\hline & & & & & & & & & & & & & & & & & & & & & & \\
\hline & & & & & & & & & & & & & & & & & & & 17 & & & \\
\hline
\end{tabular}


Tabela 7. Matriz de Adjacência gerada pelo limiar 0.75 e seus respectivos Graus.

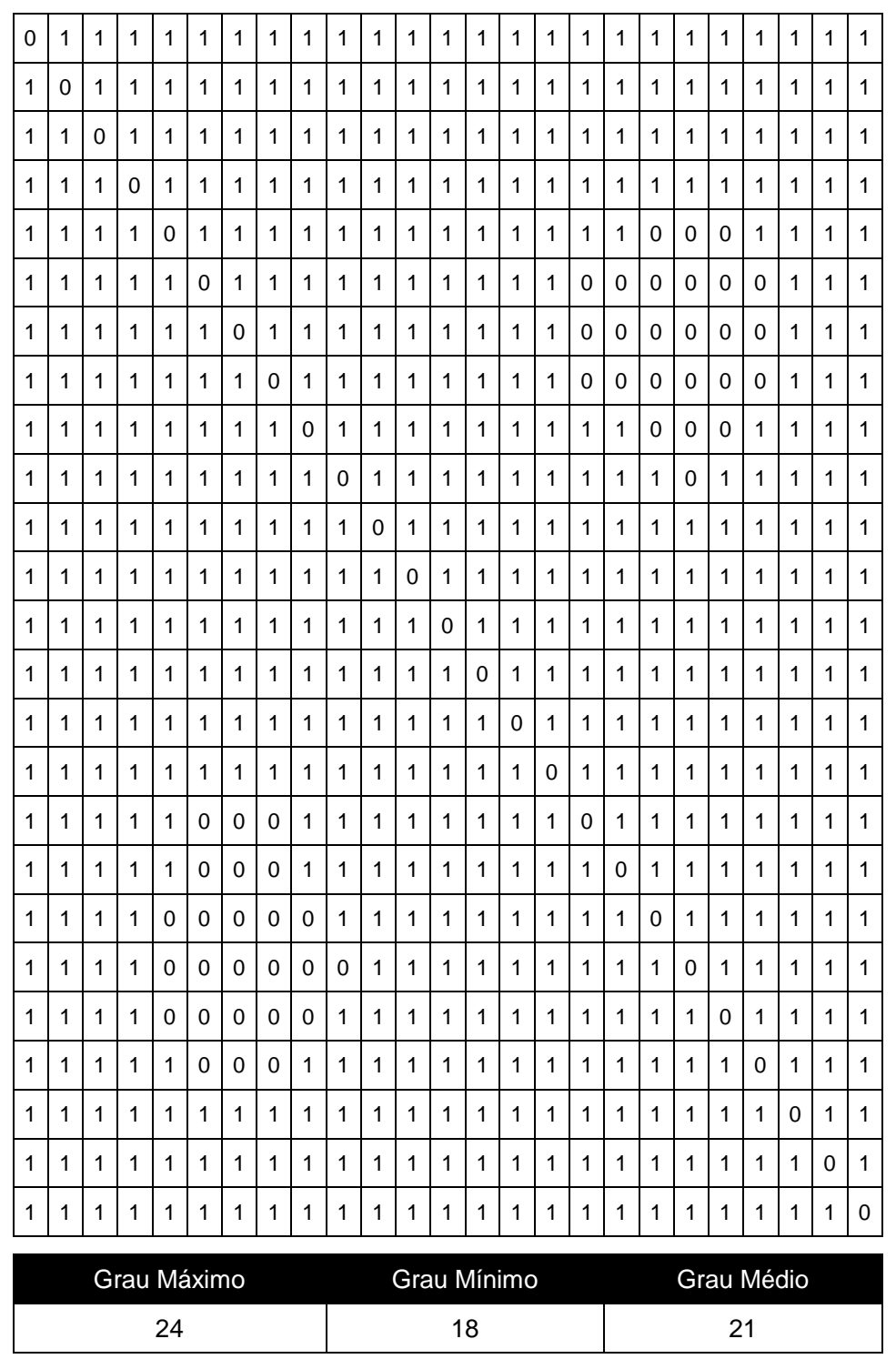


Tabela 8. Matriz de Adjacência gerada pelo limiar 0.95 e seus respectivos Graus.

\begin{tabular}{|c|c|c|c|c|c|c|c|c|c|c|c|c|c|c|c|c|c|c|c|c|c|c|c|c|c|}
\hline & 1 & 1 & 1 & 1 & & & & 1 & 1 & & & & & & & & & & & & & 1 & & & \\
\hline & 0 & 1 & 1 & 1 & & & & 1 & & & & & 1 & & & & & & & & 1 & 1 & & & \\
\hline & 1 & 0 & 1 & 1 & & & & 1 & & & & & & & & & & & & & & 1 & & & \\
\hline & 1 & 1 & 0 & 1 & & 1 & & 1 & 1 & & & & & & & & & & & & 1 & 1 & 1 & 1 & \\
\hline & 1 & 1 & 1 & 0 & & & & 1 & 1 & & & & & & & & & & & 1 & 1 & 1 & 1 & & \\
\hline & 1 & 1 & 1 & 1 & & 1 & & 1 & 1 & 1 & & & & & & & & & & & 1 & 1 & 1 & 1 & \\
\hline 1 & 1 & 1 & 1 & 1 & & 0 & & 1 & 1 & & & & & & & & & & & 0 & 1 & 1 & 1 & & \\
\hline & 1 & 1 & 1 & 1 & 1 & 1 & & 1 & 1 & 1 & & 1 & 1 & & & & & & & & 1 & 1 & 1 & 1 & \\
\hline & 1 & 1 & 1 & 1 & & 1 & & 0 & 1 & 1 & & & & & & & & & & & 1 & 1 & 1 & & \\
\hline & 1 & 1 & 1 & 1 & 1 & 1 & & 1 & 0 & 1 & & 1 & 1 & & & & & & & & 1 & 1 & 1 & 1 & \\
\hline & 1 & 1 & 1 & 1 & & 1 & & 1 & 4 & 0 & & & & & & & & & & & 1 & 1 & 1 & 1 & \\
\hline 1 & 1 & 1 & 1 & 1 & & 1 & & 1 & 1 & 1 & & 1 & 1 & & & & & & & 1 & 1 & 1 & 1 & 1 & \\
\hline 1 & 1 & 1 & 1 & 1 & 1 & 1 & & 1 & 1 & 1 & & 0 & 1 & & & & & & & 1 & 1 & 1 & 1 & 1 & 1 \\
\hline 1 & 1 & 1 & 1 & 1 & & 1 & & 1 & 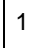 & & & & & & & & & & & 1 & 1 & 1 & 1 & 1 & 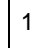 \\
\hline 1 & 1 & 1 & 1 & 1 & 1 & 1 & & 1 & 1 & 1 & & 1 & 1 & & & & & & & 1 & 1 & 1 & 1 & 1 & 1 \\
\hline 1 & 1 & 1 & 1 & 1 & & 1 & & 1 & 1 & 1 & & & & & & & & & & 1 & 1 & 1 & 1 & 1 & 1 \\
\hline . & 1 & 1 & 1 & 1 & 1 & 1 & & 1 & 1 & 1 & & 1 & 1 & & & & & & & & 1 & 1 & 1 & 1 & 1 \\
\hline 1 & 1 & 1 & 1 & 1 & 1 & 1 & & 1 & 1 & 1 & & & 1 & & & & & & & & 1 & 1 & 1 & 1 & 1 \\
\hline 1 & 1 & 1 & 1 & 1 & 1 & 1 & & 1 & 19 & 1 & & I & 1 & & & & & & & & 1 & 1 & 1 & 1 & 1 \\
\hline 1 & 1 & 1 & 1 & 1 & 1 & 0 & & 1 & 1 & 1 & & 1 & 1 & & & & & 1 & & 0 & 1 & 1 & 1 & 1 & 1 \\
\hline 1 & 1 & 1 & 1 & 1 & 1 & 1 & 1 & 1 & 1 & 1 & & 1 & 1 & & & & & & & 1 & 0 & 1 & 1 & 1 & 1 \\
\hline 1 & 1 & 1 & 1 & 1 & 1 & 1 & & 1 & 1 & 1 & & 1 & 1 & & & & & & & 1 & 1 & 0 & 1 & 1 & 1 \\
\hline 1 & 1 & 1 & 1 & 1 & 1 & 1 & 1 & 1 & 1 & 1 & & 1 & 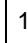 & & & & & & & 1 & 1 & 1 & 0 & 1 & 1 \\
\hline 1 & 1 & 1 & 1 & 1 & 1 & 1 & & 1 & 1 & 1 & & 1 & I & & & & & & & 1 & 1 & 1 & 1 & 0 & 1 \\
\hline & & & & 1 & & & & 1 & 1 & & & & & & & & & & & & & & & & \\
\hline
\end{tabular}

\begin{tabular}{|c|c|c|}
\hline Grau Máximo & Grau Mínimo & Grau Médio \\
\hline 24 & 23 & 23,5 \\
\hline
\end{tabular}

\section{Resultados}

Para realizar os experimentos foram utilizadas 20 imagens de peixes, onde foram extraídas suas características e selecionadas as características das bordas. Em cada imagem foram aplicados os métodos de obtenção do grau máximo, obtenção do grau mínimo e com os dois resultados, a obtenção do grau médio, este que tratará a rotação e o espelhamento de eventuais imagens similares. Para a análise e classificação das imagens, a imagem Peixe_2 foi selecionada aleatoriamente como base, onde deveremos obter as imagens que são similares a ela analisando a tolerância que foi estipulada para a seleção das imagens similares para cada limiar aplicado. Embora os resultados com limiares maiores sejam mais exatos, é interessante que haja uma diversificação nos valores dos limiares para realizar uma seleção mais detalhada das imagens. Por conta deste fator, os limiares foram selecionados aleatoriamente dentro da faixa de valores que vai de 0 a 1 , levando em consideração os resultados que constituem a matriz, presentes no mesmo intervalo, devido à normalização dos dados. Também foi definida uma variação proporcional na escolha dos valores dos limiares. Sendo assim os limiares aplicados foram $0.1,0.25,0.5,0.75$ e 0.95 . 
O limiar 0.1 é o que menos apresenta precisão nos resultados, pois permite que várias imagens sejam selecionadas dentro da faixa de tolerância. São selecionadas as imagens que possuem certos pontos com os mesmos valores de características da imagem Peixe_2. Demonstrado na Tabela 9, as imagens que obtiveram o grau médio dentro da tolerância que vai de 690 a 700 são: Peixe_2, Peixe_4, Peixe_11, Peixe_12, Peixe_14, Peixe_15 e Peixe_18.

Tabela 9. Análise para o limiar 0.1.

\begin{tabular}{|c|c|c|c|}
\hline Imagens & Máximo & Mínimo & Médio \\
\hline Peixe_1 & $\mathbf{1 2 7}$ & $\mathbf{4 5}$ & $\mathbf{8 6}$ \\
\hline Peixe_2 & 1325 & 68 & 696,5 \\
\hline Peixe_3 & 1325 & 80 & 702,5 \\
\hline Peixe_4 & $\mathbf{1 3 2 5}$ & $\mathbf{6 2}$ & $\mathbf{6 9 3 , 5}$ \\
\hline Peixe_5 & 1255 & 58 & 656,5 \\
\hline Peixe_6 & 1652 & 75 & 863,5 \\
\hline Peixe_7 & 185 & 62 & 123,5 \\
\hline Peixe_8 & 122 & 57 & 89,5 \\
\hline Peixe_9 & 1325 & 47 & 686 \\
\hline Peixe_10 & 1325 & 46 & 685,5 \\
\hline Peixe_11 & $\mathbf{1 3 2 5}$ & $\mathbf{6 8}$ & $\mathbf{6 9 6 , 5}$ \\
\hline Peixe_12 & $\mathbf{1 3 2 5}$ & $\mathbf{6 2}$ & $\mathbf{6 9 3 , 5}$ \\
\hline Peixe_13 & 1325 & 41 & 683 \\
\hline Peixe_14 & $\mathbf{1 3 2 5}$ & $\mathbf{6 2}$ & $\mathbf{6 9 3 , 5}$ \\
\hline Peixe_15 & $\mathbf{1 3 2 5}$ & $\mathbf{6 9}$ & $\mathbf{6 9 7}$ \\
\hline Peixe_16 & 1325 & 36 & 680,5 \\
\hline Peixe_17 & 1325 & 44 & 684,5 \\
\hline Peixe_18 & $\mathbf{1 3 2 5}$ & $\mathbf{6 8}$ & $\mathbf{6 9 6 , 5}$ \\
\hline Peixe_19 & 160 & 68 & 114 \\
\hline Peixe_20 & 167 & 65 & 116 \\
\hline
\end{tabular}

Utilizando o limiar 0.25, foi utilizada a mesma base de imagens, porém os resultados foram razoavelmente mais precisos, pois das sete imagens selecionadas para o limiar 0.1 , apenas cinco ficaram dentro da margem de tolerância que vai de 740 a 760 . Limiares com valor menor são utilizados como um filtro, possibilitando uma análise detalhada em cada um dos limiares através dos dados obtidos e também visualmente através das imagens especificadas neste filtro. As imagens similares no limiar 0.25 são Peixe_2, Peixe_4, Peixe_11, Peixe_14 e Peixe_15. Conforme apresenta a Tabela 10.

Tabela 10. Análise para o limiar 0.25 .

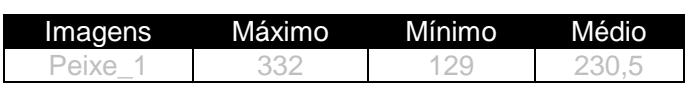

\begin{tabular}{|c|c|c|c|} 
Peixe_2 & $\mathbf{1 3 2 5}$ & $\mathbf{1 8 2}$ & $\mathbf{7 5 3 , 5}$ \\
\hline Peixe_3 & 1325 & 231 & 778 \\
\hline Peixe_4 & $\mathbf{1 3 2 5}$ & $\mathbf{1 6 5}$ & $\mathbf{7 4 5}$ \\
\hline Peixe_5 & 1255 & 162 & 708,5 \\
\hline Peixe_6 & 1652 & 208 & 930 \\
\hline Peixe_7 & 570 & 181 & 375,5 \\
\hline Peixe_8 & 370 & 156 & 263 \\
\hline Peixe_9 & 1325 & 154 & 739,5 \\
\hline Peixe_10 & 1325 & 133 & 729 \\
\hline Peixe_11 & $\mathbf{1 3 2 5}$ & $\mathbf{1 7 4}$ & $\mathbf{7 4 9 , 5}$ \\
\hline Peixe_12 & 1325 & 208 & 766,5 \\
\hline Peixe_13 & 1325 & 117 & 721 \\
\hline Peixe_14 & $\mathbf{1 3 2 5}$ & $\mathbf{1 7 5}$ & $\mathbf{7 5 0}$ \\
\hline Peixe_15 & $\mathbf{1 3 2 5}$ & $\mathbf{1 9 2}$ & $\mathbf{7 5 8 , 5}$ \\
\hline Peixe_16 & 1325 & 101 & 713 \\
\hline Peixe_17 & 1325 & 119 & 722 \\
\hline Peixe_18 & 1325 & 203 & 764 \\
\hline Peixe_19 & 420 & 190 & 305 \\
\hline Peixe_20 & 436 & 117 & 306,5 \\
\hline
\end{tabular}

O limiar 0.5 é o terceiro mais preciso, destacou uma imagem a menos dentro da faixa de tolerância. As imagens selecionadas foram Peixe_2, Peixe_4, Peixe_11 e Peixe_15, confirmadas na Tabela 11, onde a faixa de tolerância vai de 840 a 860 . Os resultados a partir deste limiar determinam as imagens que possuem características consideravelmente semelhantes, porém visualmente ainda são notadas certas diferenças.

Tabela 11. Análise para o limiar 0.5 .

\begin{tabular}{|c|c|c|c|}
\hline Imagens & Máximo & Mínimo & Médio \\
\hline Peixe_1 & 671 & 321 & 496 \\
\hline Peixe_2 & $\mathbf{1 3 2 5}$ & $\mathbf{3 8 4}$ & $\mathbf{8 5 4 , 5}$ \\
\hline Peixe_3 & 1325 & 510 & 917,5 \\
\hline Peixe_4 & $\mathbf{1 3 2 5}$ & $\mathbf{3 7 6}$ & $\mathbf{8 5 0 , 5}$ \\
\hline Peixe_5 & 1255 & 391 & 823 \\
\hline Peixe_6 & 1652 & 503 & 1077,5 \\
\hline Peixe_7 & 1011 & 412 & 711,5 \\
\hline Peixe_8 & 685 & 311 & 498 \\
\hline Peixe_9 & 1325 & 297 & 811 \\
\hline Peixe_10 & 1325 & 320 & 822,5 \\
\hline Peixe_11 & $\mathbf{1 3 2 5}$ & $\mathbf{3 8 3}$ & $\mathbf{8 5 4}$ \\
\hline Peixe_12 & 1325 & 400 & 862,5 \\
\hline Peixe_13 & 1325 & 274 & 799,5 \\
\hline Peixe_14 & 1325 & 353 & 839 \\
\hline Peixe_15 & $\mathbf{1 3 2 5}$ & $\mathbf{3 9 6}$ & $\mathbf{8 6 0}$ \\
\hline Peixe_16 & 1325 & 266 & 795,5 \\
\hline Peixe_17 & 1325 & 339 & 832 \\
\hline Peixe_18 & 1325 & 431 & 878 \\
\hline Peixe 19 & 836 & 398 & 617 \\
\hline Peixe_20 & 839 & 380 & 609,5 \\
\hline
\end{tabular}

Utilizando o limiar 0.75 , segundo mais preciso, ficaram dentro da tolerância de 940 a 960, apenas três imagens das sete que foram pré-selecionadas no limiar 0.1. Comprovada na Tabela 12, estão dentro da faixa de tolerância as 
imagens Peixe_2, Peixe_4 e Peixe_11. Assim como o limiar 0.5 , também determina as imagens que são relativamente similares. Mais exato, porém ainda exibe algumas inconformidades visuais quanto à comparação entre a imagem Peixe_2 e as imagens selecionadas Peixe_4 e Peixe_11.

Tabela 12. Análise para o limiar 0.75 .

\begin{tabular}{|c|c|c|c|}
\hline Imagens & Máximo & Mínimo & Médio \\
\hline Peixe_1 & 684 & 514 & 599 \\
\hline Peixe_2 & 1325 & 569 & 947 \\
\hline Peixe 3 & 1325 & 774 & 1049,5 \\
\hline Peixe_4 & 1325 & 579 & 952 \\
\hline Peixe_5 & 1255 & 598 & 926,5 \\
\hline Peixe 6 & 1652 & 756 & 1204 \\
\hline Peixe 7 & 1019 & 749 & 884 \\
\hline Peixe 8 & 689 & 494 & 591,5 \\
\hline Peixe 9 & 1325 & 604 & 964,5 \\
\hline Peixe_10 & 1325 & 480 & 902,5 \\
\hline Peixe_11 & 1325 & 565 & 945 \\
\hline Peixe 12 & 1325 & 606 & 965,5 \\
\hline Peixe 13 & 1325 & 421 & 873 \\
\hline Peixe_14 & 1325 & 539 & 932 \\
\hline Peixe 15 & 1325 & 654 & 989,5 \\
\hline Peixe 16 & 1325 & 440 & 882,5 \\
\hline Peixe 17 & 1325 & 490 & 907,5 \\
\hline Peixe_18 & 1325 & 704 & 1014,5 \\
\hline Peixe_19 & 840 & 609 & 724,5 \\
\hline Peixe 20 & 843 & 617 & 730 \\
\hline
\end{tabular}

O limiar 0.95 é o último limiar a ser analisado e também o mais preciso entre os cinco estabelecidos. Este limiar apresenta os resultados finais do experimento. Dentre todas as imagens analisadas, a que mais se aproximou da similaridade referente à imagem Peixe_2 foi a imagem Peixe_11, pois ambas ficaram na faixa de tolerância que vai de 1020 a 1040, conforme exibe a Tabela 13.

Tabela 13. Análise para o limiar 0.95 .

\begin{tabular}{|c|c|c|c|}
\hline Imagens & Máximo & Mínimo & Médio \\
\hline Peixe 1 & 684 & 637 & 660,5 \\
\hline Peixe_2 & 1325 & 744 & 1034,5 \\
\hline Peixe 3 & 1325 & 1020 & 1172,5 \\
\hline Peixe 4 & 1325 & 771 & 1048 \\
\hline Peixe 5 & 1255 & 773 & 1014 \\
\hline Peixe 6 & 1652 & 980 & 1316 \\
\hline Peixe 7 & 1019 & 963 & 991 \\
\hline
\end{tabular}

\begin{tabular}{|c|c|c|c|} 
Peixe_8 & 689 & 645 & 667 \\
\hline Peixe_9 & 1325 & 834 & 1079,5 \\
\hline Peixe_10 & 1325 & 595 & 960 \\
\hline Peixe_11 & $\mathbf{1 3 2 5}$ & $\mathbf{7 4 1}$ & $\mathbf{1 0 3 3}$ \\
\hline Peixe_12 & 1325 & 807 & 1066 \\
\hline Peixe_13 & 1325 & 528 & 926,5 \\
\hline Peixe_14 & 1325 & 699 & 1012 \\
\hline Peixe_15 & 1325 & 861 & 1093 \\
\hline Peixe_16 & 1325 & 576 & 950,5 \\
\hline Peixe_17 & 1325 & 645 & 985 \\
\hline Peixe_18 & 1325 & 940 & 1132,5 \\
\hline Peixe_19 & 840 & 794 & 817 \\
\hline Peixe_20 & $\mathbf{8 4 3}$ & $\mathbf{8 0 1}$ & $\mathbf{8 2 2}$ \\
\hline
\end{tabular}

\section{Discussão}

A análise de imagens se tornou um desafio para os pesquisadores da área de visão computacional por ter o objetivo de reconhecer formas do mundo real. Para obter resultados mais concretos, os métodos das Redes Complexas foram utilizados para dar eficiência nas comparações de similaridade entre as imagens. $\mathrm{O}$ objetivo dos experimentos foi provar que extraindo o grau máximo, mínimo e médio, e aplicando um determinado limiar a este, um resultado preciso é adquirido, em que quanto maior o valor do limiar, maior será a precisão nos resultados. Fazendo uso de cinco limiares diferentes, no limiar 0.95 foi encontrada a similaridade entre as imagens Peixe_2 e Peixe_11.

\section{Referências}

AIELLO, W.; CHUNG, F.; LU, L. A. Random Graph Model for Massive Graphs. Proceedings of 32nd Annual ACM Symposium on Theory of Computing, Association of Computing Machinery. New York. 2000. p.171-180.

ALBERT, R.; BARABÁSI, A. L. Emergence of Scaling in Random Networks. Science. v.286, p.509-512, 1999.

http://dx.doi.org/10.1126/science.286.5439.509

BERRETTI, S.; DEL BIMBO, A.; VICARIO, E. Efficient Matching and Indexing of Graph Models in Content-Based Retrieval. IEEE Transactions on Pattern Analysis and Machine Intelligence, v.23, 
n.10, $\quad 2001$. http://dx.doi.org/10.1109/34.954600

COSTA, L. F.; CESAR, R. M. Shape Analysis and Classification: Theory and Practice. Pennsylvania: CRC Press, 2000. http://dx.doi.org/10.1201/9781420037555

FALCÃO, A. X.; STOLFI, J.; LOTUFO, R. A. The image foresting transform: Theory, algorithms, and applications. IEEE Transactions on Pattern Analysis and Machine Intelligence, v.26, n.1, p.1929 , 2004. http://dx.doi.org/10.1109/TPAMI.2004.1261076

JAIN, A. K. Fundamentals of Digital Image Processing. London: Prentice Hall, 1989.

MATHIAS, E.; CONCI, A. Pesquisa de Imagens em Banco de Dados por Semelhança de Cor. In: X Simpósio Brasileiro de Computação Gráfica e Processamento de Imagens, Campos do Jordão, SP, Out. 1997.

BOAVENTURA NETTO, P. O. Grafos: Teoria, Modelos, Algoritmos. 3.ed. Ed. Edgard Blücher. 2003. 314p.

NEWMAN, M. E. J. The Structure and Function of Complex Networks. SIAM Review. v.45, n.2, p.167-256, 2003. http://dx.doi.org/10.1137/S003614450342480

SCHWARTZ, W. R.; PEDRINI, H. Método para Classificação de Imagens Baseada em Matrizes de Co-ocorrência Utilizando Características de Textura. In: Proceedings of III Colóquio Brasileiro de Ciências Geodésicas, Maio 2003. p.1-11.

SCLAROFF, S.; TAYCHER, L.; CASCIA, M. ImageRover: A Content-Based Image Browser for the World Wide Web. In: Proceedings IEEE Workshop on Content-based Access if Image and Video Libraries, EUA: 1997.

SONKA, M.; HLAVAC, V. et al. Image Processing Analysis, and Machine Vision. Pacific Grove: PWS Publishing, 1999. $770 \mathrm{p}$.
ULLMANN, J. R. An Algorithm for Subgraph Isomorphism. Journal of the ACM, v.23, p.31-42, 1976. http://dx.doi.org/10.1145/321921.321925

RABUSKE, M. A. Introdução à Teoria dos Grafos. Editora da UFSC, Santa Catarina, 1992.

MOKHTARIAN, F.; ABBASI, S.; KITTLER, J. Robust and efficient shape indexing through curvature scale space, in Proceedings of the 1996 British Machine and Vision Conference BMVC'96, Edinburgh, U.K., pp. 53-62, 1996.

BACKES, A. R.; CASANOVA, D.; BRUNO, O. M. Journal of Computer Science, v. 6, n. 2, p.71-80, 2007. 